\title{
Concurrent Session 2
}

\section{ACP and Chronic Illness}

\section{A COLLABORATIVE ENGAGEMENT OF ADVANCE CARE PLANNING WITHIN A DIVERSE CHRONIC DISEASE POPULATION}

D Murphy-Burke1, W Robinson ${ }^{2}{ }^{1}$ British Columbia Provincial Renal Agency; 2Providence Health Care

10.1136/bmjspcare-2012-000250.29

Patients living with Chronic Kidney Disease (CKD) have a high symptom burden associated with multi-co morbidities including diabetes and cardiovascular disease. The annual mortality rate of CKD patients is $25 \%$, most of whom have not had the opportunity to engage in Advance Care Planning (ACP). This reality spurred on the British Columbia Provincial Renal Agency (BCPRA) to engage dedicated renal leaders from within BC to develop an 'End of Life Framework' for renal patients. This framework includes guidelines and recommended steps to address ACP with renal patients within B.C.'s 5 geographic Health Authorities (HA). We will describe the collaborative development of this framework and the subsequent implementation of a systematic, interdisciplinary approach to ACP within a culturally diverse 275 patient, haemodialysis unit at St. Paul's Hospital, Vancouver. We will share the successes as well the obstacles and mitigation strategies of implementation, from initial planning, program engagement, comprehensive staff education through to a pilot using the My Voice document and evaluation. We will share how ACP protocols are now embedded in the practice of a core interdisciplinary ACP team. First year audit results that indicate a marked improvement to a minimum $40 \%$ of haemodialysis patients having documentation of ACP conversations will be discussed. We will describe how the learnings from the ACP implementation at this renal unit are being shared and applied in diverse renal settings across this province. 